\title{
INNOVACIÓN Y TECNOLOGÍA EN EL TERCER SECTOR: PARADIGMAS Y DESAFÍOS
}

\section{INNOVATION AND TECHNOLOGY IN THE THIRD SECTOR: PARADIGMS AND CHALLENGES}

\author{
MSC@ Carlos Alberto Pacheco Sánchez*, MSC. Belén Torcoroma Quintero \\ Bayona $^{* *}$ MSC@. Leidy Torcoroma Guerrero Prado*** Edward Fabián Moreno \\ Mendoza*****
}
* Universidad Francisco de Paula Santander Ocaña, Facultad de Ciencias Administrativas y Económicas, Administración de Empresas, Grupo de Investigación GIDSE.
Vía Acolsure, Sede el Algodonal, Ocaña, Norte de Santander, Colombia. (+57) (7) 5690088 - Fax: Ext. 104,
E-mail: \{capachecos, btquinterob, ltguerrerop, efmorenom $\} @$ ufpso.edu.co,

Resumen: La evolución de las tecnologías en el tercer sector de la economía imponen retos y a su vez direccionan las dinámicas administrativas a la búsqueda de nuevos modelos y mejores sistemas de información, por tanto, el propósito del estudio se centra en conocer la situación actual de las organizaciones solidarias con relación a los procesos de innovación tecnológica, de igual manera, identificar los desafíos corporativos de las mismas para lograr sustentabilidad y por ultimo plantear estrategias de tipo tecnológico con el fin de apoyar la dirección de los recursos en función de sus principios y fines solidarios. Se concluye que los líderes del sector solidario del municipio de Ocaña presenta desconocimiento, paradigmas, barreras y altos grados de incertidumbre frente a la imposición de sistemas tecnológicos y en su efecto llevan procesos tan tradicionales, burocráticos y ambiguos que le apuestan al escepticismo e idealismo frente al mundo digital.

Palabras claves: Desafíos; Estrategias; Innovación; Organizaciones solidarias; TIC's Abstract: The evolution of the technologies in the third sector of the economy impose challenges and at the same time direct the administrative dynamics to the search of new models and better information systems, therefore, the purpose of the study is centered in knowing the current situation of the solidary organizations in relation to the processes of technological innovation, of equal way, to identify the corporative challenges of the same ones to achieve sustainability and finally to propose strategies of technological type in order to support the direction of the resources in function of its principles and solidary aims. It is concluded that the leaders of the solidarity sector of the municipality of Ocaña presents ignorance, paradigms, barriers and high degrees of uncertainty in front of the imposition of technological systems and in their effect they take processes so traditional, bureaucratic and ambiguous that they bet on skepticism and idealism in front of the digital world.

Keywords: Challenges; strategies; innovation; Solidarity organizations; TIC's. 


\section{INTRODUCCIÓN}

El tercer sector de la economía colombiana, impulsa día a día diferentes acciones en pro del bienestar de una comunidad, sin embargo, muchas de ellas a pesar de buscar los beneficios sociales, la integración humana de sus asociados, la autogestión de los procesos y la preservación del medio ambiente, presentan dificultades en la imposición de tecnologías y estrategias innovadoras que no solo afectan los esquemas organizacionales sino también productivos, debido a que, desde las direcciones existe el propósito principal de anteponer los objetivos corporativos y superar las expectativas de sus stakeholders primarios y secundarios a través de su compromiso social; desde esta perspectiva, (Orrego y Arboleda, 2006) hacen alusión a los diferentes paradigmas de gestión estratégica que han existido y que sin duda alguna siguen teniendo presencia en las organizaciones sin ánimo de lucro, a manera de ejemplo, los entes solidarios en la actualidad, independientemente de su reconocimiento axiológico, se han preocupado por tratar de asegurar la sostenibilidad, enfocándose en alcanzar la productividad y la optimización de sus recursos (Paradigmas clásicos y neoclásicos), por otra parte, las mismas se sustentan en promover la unión y los cambios positivos en la conducta de nuestra sociedad tratando por lo general de solventar los criterios de satisfacción y de autorrealización, lo anterior, resulta coherente con los fines y valores asociativos, no obstante, muy pocas han logrado romper el paradigma cuantitativo, de integración y de cultura, lo que limita a que puedan ser flexibles en el flujo de información y en los procesos principales que dan cumplimiento a sus actividades económicas, entonces, la toma de decisión se ve afectada por los constantes cambios de la globalización y por el alto grado de preocupación e incertidumbre por tratar de responder cuáles son los avances tecnológicos e innovadores más apropiados para alterar positivamente los sistemas interdependientes a su objeto.

Ahora bien, cuando las organizaciones solidarias con muchos años de funcionamiento y con esquemas de trabajo muy tradicionales, despiertan el interés por introducir capital tecnológico básico que apoye y agilice ciertos procedimientos elementales del órgano empresarial, algunas desconfían y/o consideran que lo principal por atender son los objetivos corporativos, por tanto, el desarrollo de la investigación permite reconocer las condiciones actuales que presenta el tercer sector económico con relación a la implementación de las TIC's, así mismo, la identificación de los desafíos tecnológicos e innovadores que buscan imponer en el tiempo con fines de llevar a cabo mejores acciones (administrativas, legales, tributarias, económicas y operativas) propias en la gestión impositiva de sus principales directivos. Adicional a ello, se formulan y se propone al sector solidario de Ocaña, Norte de Santander, estrategias que coadyuven al diseño de nuevos planes y programas centralizados a dinamizar sus subsistemas con herramientas tecnológicas claves para atender sus necesidades y para generar ventajas competitivas, rompiendo barreras que impiden su crecimiento a nivel nacional e internacional.

\section{MARCO TEÓRICO}

Las organizaciones del sector solidario han entendido que los avances y los procesos de tecnología y de innovación siempre actuarán en función de los contextos y las condiciones administrativas de una organización, es así como prevalece el interés constante de las gerencias por evaluar las estructuras internas, de tal manera que se pueda reconocer qué acciones edifican grandes cambios, sin alterar tanto las conductas del capital humano o de sus recursos tangibles, esto con el propósito de evitar caos ante las distintas actividades que ejecuta la entidad. A continuación se apropia el constructo científico establecido para el proceso investigativo:

\subsection{Teoría organizacional}

La teoría a pesar de ser clásica, se fundamenta en la estructura interna de las organizaciones, con el fin de obtener resultados satisfactorios que generen un beneficio no solo para la organización, sino también a todo el personal que la conforma; pues por medio de esta, es posible demostrar la importancia que tiene la dirección en la ejecución de todos los procesos y actividades, para su desarrollo (Rivas, 2009). Así mismo, es una teoría que hace un acercamiento macro del comportamiento de las empresas, de hecho, permite evidenciar la complejidad que tienen las organizaciones en su contexto, indicando, que es necesario que las actividades de mejoramiento propuestas sean aplicadas en varios niveles: individual, interpersonal, por grupos e inter grupos, en síntesis, se puede considerar que la teoría organizacional, promueve el cambio planeado a pesar de sus intervenciones, en donde la colaboración de los diferentes niveles organizacionales hacen posible que las mismas puedan lograr crecimiento, desarrollo y rentabilidad (Rivas, 2009). 


\subsection{Modelo de Aceptación de Tecnología (TAM)}

Durante las últimas décadas, diversos autores han centrado sus estudios respecto al uso y aceptación de las nuevas tecnologías. El Modelo de la Aceptación de Tecnología, tiene una gran influencia por la teoría de Acción Razonada de Fishbein y Ajzen, lo relevante de este modelo, recae en la explicación de los componentes del comportamiento para la aceptación de la tecnología, basándose en la facilidad de uso y la utilidad percibida por los usuarios para predecir el uso de la tecnologías (Yong, Rivas y Chaparro, 2010).

\subsection{Innovación administrativa e Innovación técnica}

La innovación organizacional es gestionada como consecuencia de los efectos que se esperan a partir de los nuevos procesos que puedan generarse, no obstante, es importante evaluar los enfoques que presenta dicha innovación. Incluso Damanpour y colaboradores (1989) sustentan que para mantener o mejorar el nivel de desempeño, es necesaria la adopción balanceada de la innovación administrativa y la innovación técnica haciendo a la organización más efectiva, teniendo en cuenta que las innovaciones técnicas promueven la eficiencia organizacional, y que las innovaciones administrativas son las que obtienen un balance general entre la estructura social y el sistema técnico de la organización (Yamakawa y Ostos, 2011).

Tabla 1: Tipos de innovación

\begin{tabular}{|c|c|}
\hline $\begin{array}{c}\text { Tipo de } \\
\text { innovación }\end{array}$ & Concepto \\
\hline $\begin{array}{l}\text { Innovación } \\
\text { de producto }\end{array}$ & $\begin{array}{l}\text { Aporta un bien o un servicio nuevo, } \\
\text { mejorando sus características, técnicas, } \\
\text { uso u otras funcionalidades. }\end{array}$ \\
\hline $\begin{array}{l}\text { Innovación } \\
\text { de proceso }\end{array}$ & $\begin{array}{l}\text { Cambios significativos en las técnicas, } \\
\text { los materiales y/o los programas } \\
\text { informáticos empleados en la } \\
\text { organización. }\end{array}$ \\
\hline $\begin{array}{l}\text { Innovación } \\
\text { en } \\
\text { Marketing }\end{array}$ & $\begin{array}{l}\text { Consiste en utilizar un método de } \\
\text { comercialización. }\end{array}$ \\
\hline $\begin{array}{l}\text { Innovaciones } \\
\text { organizativas }\end{array}$ & $\begin{array}{l}\text { Cambios en las prácticas } \text { y } \\
\text { procedimientos, modificaciones en el } \\
\text { lugar de trabajo y en las relaciones } \\
\text { exteriores. }\end{array}$ \\
\hline
\end{tabular}

Fuente: Adaptación del Manual de Oslo, p.58-60 citado por (Jansa, 2010, pág. 5).

Es ineludible destacar que los distintos procesos innovadores de las organizaciones se enmarcan desde las realidades que vive el tejido empresarial, por ello, para los próximos años las innovaciones tienen como prioridad abarcar y atender clientes por medio de las tendencias del e-commerce, tras lo anterior, el mercado demandante espera procesos de compra, de comunicación y de atención más sencillos, confiables, prácticos y flexibles, es decir, una aproximación a la humanidad digital.

\subsection{Teoría de innovación organizacional}

Según Lam (2004) (citado por Arraut, 2008, p.188), establece que la innovación organizacional hace referencia a las siguientes áreas de estudio:

1. La innovación en sí. 2. Los diferentes tipos de innovación en las organizaciones. 3. Los cambios en la estructura de la organización.

Ahora bien, es de suma importancia comprender que la innovación desde el campo administrativo imparte cambios a partir de la estructura conforme a los procesos que emplea, de hecho, la innovación organizacional se caracteriza por la búsqueda de nuevos diseños que alteran las estructuras internas, implicando cambios entre los límites de la organización y el contexto. De acuerdo con Hölzl, Reinstaller y Windrum (2005, p.4), estos nuevos diseños están basados en la teoría modular la cual fracciona las labores administrativas con el fin de mejorar el control de cada uno de los elementos modulares y la interacción entre los componentes de la organización (Arraut, 2008).

\section{METODOLOGÍA}

La investigación es un estudio descriptivo con enfoque cuantitativo, que de acuerdo a lo mencionado por (Behar, 2008) sirven para analizar cómo es y cómo se manifiesta un fenómeno y sus componentes a través de la medición de uno o más de sus atributos. Por consiguiente, con el método inductivo-deductivo se definió un diseño probabilístico, donde la población de estudio estuvo conformada por 100 organizaciones solidarias activas en el territorio local, seleccionadas a través del muestreo aleatorio simple; aplicando la técnica de la encuesta a directivos, gerentes o administradores de dichas organizaciones. Seguidamente, se realizó un respectivo análisis estadístico cuantitativo (Síntesis estadístico) y cualitativo para presentar propiedad en las conclusiones teniendo en cuenta los aportes de (Dávila, 2006) quien manifestó la importancia de establecer conclusiones generales basándose en hechos recopilados mediante observaciones y verdaderas premisas. 


\section{RESULTADOS}

Al analizar los datos primarios obtenidos por el instrumento, se hace un reconocimiento de tres tipos de innovaciones más importantes en las organizaciones solidarias de Ocaña, de igual forma, una evaluación de los desafíos que presentan las mismas a pesar de las barreras o paradigmas existentes en sus estructuras, considerando que hoy la sociedad de la información y del conocimiento ha llevado a que el reto principal es aplicar y dar respuesta a los cambios mediante las TIC's, siendo en ultimas, un compromiso para las entidades sin ánimo de lucro, ya que no solo deben promover actividades que den respuesta o agilice la entrega de información a órganos públicos, privados o de vigilancia, sino que también puedan contribuir al desarrollo de la economía y de las condiciones sociales de nuestro territorio, acotando que la tecnología y la innovación transforma, esquematiza y mejora diferentes acciones gerenciales en las organizaciones estudiadas.

\section{Tabla 2. Innovaciones en el Tercer Sector}

Fuente: Elaboración propia.

Es evidente que la aplicación de las tecnologías de la información y la comunicación (TIC's) simplifica tareas tediosas, con el propósito de poder atender y dar respuesta a las funciones administrativas más complejas, tratando de llevar un proceso exhaustivo que pueda evaluar y mediar los rendimientos, pero sobre todo, generar valor agregado a los diferentes campos de acción (Zapata, Arango y Adarme, 2010). Frente a lo planteado y partiendo de los resultados alcanzados, se sustenta que las organizaciones deben estar enfocadas a manejar, crear o implementar programas, plataformas, aplicativos y espacios desde la virtualidad para tratar de alcanzar los desafíos y retos propuestos, de hecho, se deduce que muchas organizaciones del tercer sector están en la necesidad de implementar sistemas de información capaces de automatizar actividades que refuercen los procesos y la comunicación asíncrona y síncrona dentro de ellas.

A continuación se presentan los datos sobre las tecnologías más importantes que deben implementar las organizaciones solidarias de acuerdo a los desafíos y seguidamente se proponen estrategias.
Tabla 3. Acciones tecnológicas de carácter comunicativo en las organizaciones de economía solidaria

\begin{tabular}{ccc}
\hline $\begin{array}{c}\text { Tipos de } \\
\text { herramientas } \\
\text { tecnológicas }\end{array}$ & $\begin{array}{c}\text { Barreras o } \\
\text { Paradigmas }\end{array}$ & Desafíos \\
\hline
\end{tabular}




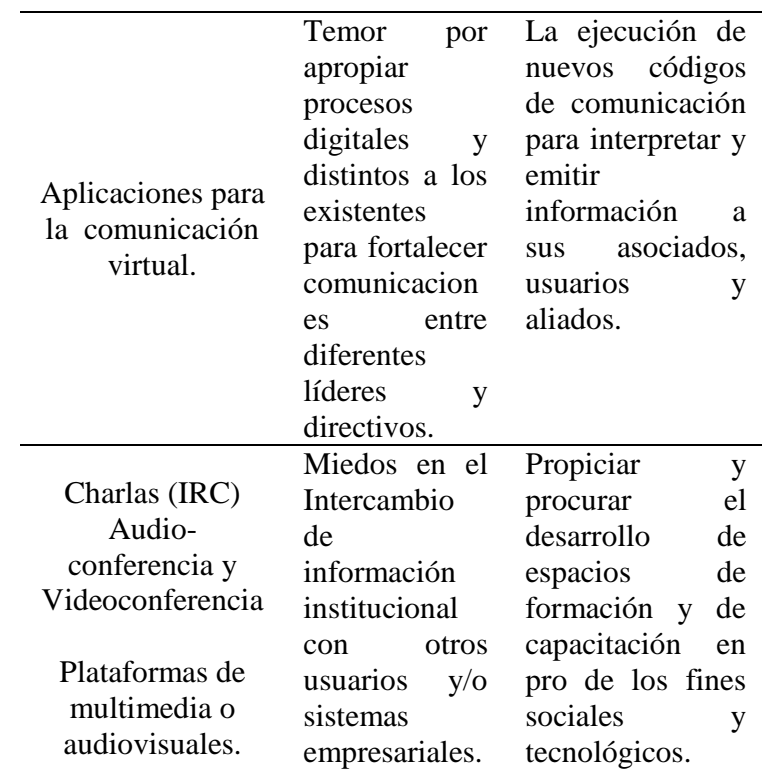

Fuente: Elaboración propia.

Estrategias para las acciones tecnológicas de carácter comunicativo en las organizaciones de economía solidaria

Establecer políticas para dinamizar la comunicación asincrónica.

Diseñar la planificación de recursos empresariales en función de las TIC's

Intercambiar mensajes entre usuarios de ordenadores conectados a Internet.

Construir mensajería instantánea: Correo electrónico institucional, WhatsApp, Hangouts, Messenger, Snapchat, Line, Skype, Vibre, entre otros.

Desarrollar protocolos de Comunicación sincrónica a través de capacitaciones o talleres en:

Aplicar buscadores: Google Chrome, Internet Explorer, Mozilla Firefox, Opera Mini, iCab, OmniWeb.

Organizar documentos en la nube: Dropbox, Google Drive, iCloud, WeTransfer, Jumpshare.

Desarrollar espacios informativos: Grupos de noticias o foros de debate (Newsgroups) con diferentes tablones de anuncios, donde cualquier usuario puede enviar su comentario, respuesta o apreciación del evento.

Tabla 4. Acciones tecnológicas de carácter operativo en las organizaciones de economía $\underline{\text { solidaria }}$

\begin{tabular}{ccc}
\hline $\begin{array}{c}\text { Tipos de } \\
\text { herramientas } \\
\text { tecnológicas }\end{array}$ & $\begin{array}{c}\text { Barreras o } \\
\text { Paradigmas }\end{array}$ & Desafíos \\
\hline
\end{tabular}

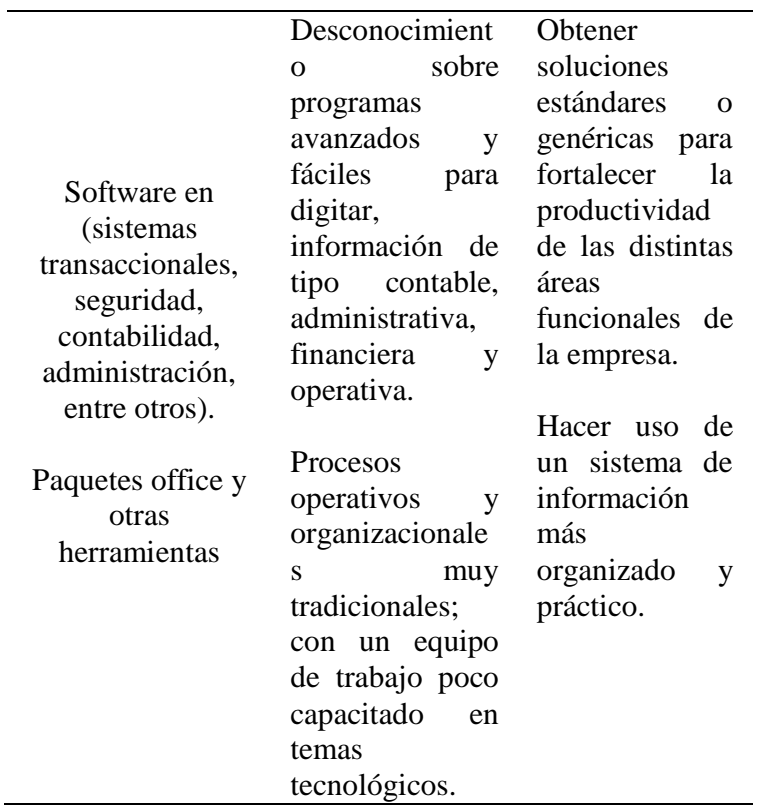

Fuente: Elaboración propia.

Estrategias para acciones tecnológicas de carácter operativo en las organizaciones de economía solidaria

Crear y desarrollar un modelo de sistema de información compatibles con sus necesidades, procesos, actividades y tareas a través del apoyo y acompañamiento de otras instituciones.

Construir datas y redes de información con otras organizaciones para hacer replica de las actividades institucionales en especial sobre las prácticas sociales y la actualización de procesos en el marco de la normativa.

Tabla 5. Acciones tecnológicas para la optimización y presentación de información electrónica

\begin{tabular}{|c|c|c|}
\hline $\begin{array}{c}\text { Tipos de } \\
\text { herramientas } \\
\text { tecnológicas }\end{array}$ & $\begin{array}{c}\text { Barreras o } \\
\text { Paradigmas }\end{array}$ & Desafíos \\
\hline $\begin{array}{l}\text { Páginas web } \\
\text { (World Wide } \\
\text { Web -www). }\end{array}$ & $\begin{array}{l}\text { Incertidumbre } \\
\text { para ofrecer } \\
\text { información } \\
\text { propia y de } \\
\text { calidad por } \\
\text { parte de la } \\
\text { organización, a } \\
\text { nivel nacional e } \\
\text { internacional. } \\
\text { Escaso } \\
\text { financiamiento } \\
\text { para de } \\
\text { desarrollo de } \\
\text { aplicativos web. }\end{array}$ & $\begin{array}{lr}\text { La utilización de } \\
\text { técnicas para la } \\
\text { búsqueda } & \text { y } \\
\text { entrega } & \text { de } \\
\text { información } & \\
\text { efectiva. } & \\
\text { Organizar ros } \\
\text { sistemas lo } \\
\text { información para } \\
\text { el desarrollo de } \\
\text { los diferentes } \\
\text { procesos. }\end{array}$ \\
\hline
\end{tabular}


Fuente: Elaboración propia.

\section{Estrategias para la optimización y presentación de información electrónica}

Generar procesos de formación y capacitación sobre el manejo y utilización de información teniendo en cuenta las TIC's y sitios web.

Desarrollar CRM para gestionar la relación con los clientes a través de un proceso de

interconectividad.

Las anteriores estrategias se definen con el propósito de fomentar en las entidades del tercer sector la aplicación de sistemas digitales donde permita desarrollar e integrar diversos tipos de procesos de información y de comunicación, para ello, es pertinente que las organizaciones en estudio realicen intercambios de datos en tiempo real o atemporal aplicando el siguiente referente:

La comunicación sincrónica es el intercambio de información por Internet en tiempo real y la comunicación asincrónica se establece entre dos o más personas de manera diferida en el tiempo, es decir, cuando no existe coincidencia temporal. (Lamí, Pérez y Rodríguez, 2016, p. 85)

Ahora bien, al dar cumplimiento de lo mencionado el tercer sector permite que los sistemas organizacionales puedan desarrollar esquemas de planificación y de flujos de información más completos e interdisciplinarios, sin dejar de lado, el diseño de un modelo apropiado, que pueda dar respuesta a las expectativas que tengan los altos directivos, empleados, asociados o comunidad en general (Stakeholder).

\section{CONCLUSIONES}

Los cambios y avances tecnológicos ha llevado a las organizaciones solidarias a adjudicarse a distintos escenarios a los que existían en décadas anteriores, dado a que el reto de toda empresa, es asumir los cambios de la globalización, realizando innovación a través de las herramientas tecnológicas con el fin de ejecutar ejercicios administrativos sistematizados y automatizados asegurando acciones factibles.

De hecho, se entiende que desde nuestros territorios muchos emprendedores y empresarios han adquirido hardware y software para adaptarse y adoptar los nuevos procesos tecnológicos e innovadores acorde a las exigencias de otras organizaciones públicas y privadas, sin embargo, han tenido barreras, errores e inconvenientes de carácter ordinario o extraordinario a causa de actividades ambiguas, burocráticas y tradicionales, tan así que Goldsby y Martichenko citado por Zapata, et al. (2010) argumentan lo indispensable que es organizar las tres áreas estratégicas donde se centran los flujos de información (datos, comunicación y conocimiento) pero la tarea termina siendo difícil para ellas, debido a los paradigmas originados por las denominadas Nuevas Tecnologías de la Información y la Comunicación (NTIC), pues están suscitando más retos y desafíos a las organizaciones, con el propósito de que ellas puedan acceder al enorme potencial de información existente, al creciente papel de las innovaciones digitales, al aumento continuo de la demanda de información y nuevos conocimientos y a la tendencia de comercialización en una amplia red de telecomunicaciones, que podrán ser ejecutadas por tres componentes esenciales: La información, la comunicación y la tecnología que resultan claves en la estructura y el funcionamiento de la sociedad, dado a su alcance, grado de influencia y penetración en los distintos sectores y niveles de la vida social (Cabada, 2001).

Si bien es claro que, en el mundo del tercer sector se están generando grandes desarrollos y uno de los componentes principales en este, son el uso de las TIC's, estableciéndolas como uno de los elementos de mayor importancia para el cumplimiento de los objetivos organizacionales y sumado a ello, se ha convertido en un pilar clave e indispensable para aquellos organismos que las vigilan, ya que se les facilita las tareas de recolección y supervisión de diferentes datos, tanto así, que muchas hacen redireccionamiento de ciertos procesos según las normativas, por eso, las organizaciones solidarias a la fecha deben contar con las herramientas tecnológicas y los Sistemas de Administración de Riesgo de Lavado de Activos y Financiación del Terrorismo (SARLAFT) conforme a lo establecido en la circular externa $\mathrm{N}^{\circ}$ 04: Capturar, validar y actualizar periódicamente la información; consolidar las operaciones; generar en forma automática los reportes internos y externos, y que todos los reporte de la UIAF sean enviados de forma electrónica (Superintendencia de la economía solidaria, 2017). De igual manera, sucede con los lineamientos establecidos en el Decreto 2150, donde las entidades no contribuyentes del régimen tributario especial, requieren precisar y suministrar información en el registro o aplicativo que establece la DIAN, con el objeto de dar transparencia al proceso de calificación, permanencia y actualización 
presentadas mediante memorias económicas digitales (artículo 364-5 del estado tributario) (Ministerio de Hacienda y Crédito Público, 2017). Siendo lo anterior, para muchas asociaciones, fundaciones, fondos, cooperativas y demás entes, una gran barrera y una problemática difícil de atender como consecuencia del poco conocimiento de cuáles actividades y qué procesos tecnológicos tienen que ejecutar para dar respuesta a lo establecido en el Decreto, entonces, las estrategias planteadas tienen como fin promover las acciones de planificación, implementación, seguimiento y retroalimentación de la dinámica que surgen del acelerado proceso de la humanidad digital.

Finalmente, cuando se da uso de las estrategias tecnológicas y se fomenta la innovación, se puede afirmar que el mundo digital sin importar las clasificaciones que se designen, ayuda a que las organizaciones con fines sociales, propongan $\mathrm{y}$ formen exitosos modelos de negocios, tal como lo propone (Berisha, 2015) en su estudio Impact of Information Technology and Internet in Businesses, pues al analizar los hallazgos de ambas investigaciones se determina que cuando las TIC's son implementadas y evaluadas de acuerdo a las tendencias globales, hace que las organizaciones en gran medida aseguren desarrollo y crecimiento, pero sobre todo, puedan afrontar la economía del siglo XXI con una visión universal y sin brechas digitales.

\section{REFERENCIAS}

Arraut, L. (2008). La Innovación de Tipo Organizacional en las Empresas Manufactureras de Cartagena de Indias. Semestre Económico, Vol. 11, No. 22. $(185-203)$.

Behar, D. (2008). Metodología de la Investigación. Editorial Shalom.

Berisha, A. (2015). Impact of Information Technology and Internet in Businesses. Academic Journal of Business, Administration, Law and Social Sciences, Vol. 1, No. 1, (73-79).

Cabada, M. (2001). Estudio del paradigma tecnológico y su repercusión en la formación de los profesionales de la información. Revista ACIMED, Vol. 9, No. 3. (224-228).

Dávila, G. (2006). El razonamiento inductivo y deductivo dentro del proceso investigativo en ciencias experimentales y sociales. Revista de Educación, Vol. 12. (180-205).
Jansa, S. (Septiembre de 2010). RESUMEN DEL MANUAL DE OSLO SOBRE INNOVACIÓN. https://bit.ly/15WZDO2. (Consultado: 29 de Agosto de 2018)

Lamí, L., Pérez, M., \& Rodríguez, M. (2016). Las herramientas de comunicación sincrónica y asincrónica en la clase presencial. Revista pedagógica de la Universidad de Cienfuegos, Vol. 12, No.12. (84-89).

Ministerio de Hacienda y Crédito Público. (20 de Diciembre de 2017). Decreto 2150. https://bit.ly/2CBE6i6. (Consultado: $15 \mathrm{de}$ Agosto de 2018)

Orrego, C., \& Arboleda, A. (2006). Las organizaciones de economía solidaria: Un modelo de gestión innovador. Cuadernos de administración (34), 97-110.

Rivas, L. (2009). Evolución de la teoría de la organización. Universidad y empresa, Vol. 11, No. 17. (11-32).

Saavedra, M., \& Tapia, B. (2013). El uso de las tecnologías de información y comunicación TIC en las micro, pequeñas y medianas empresas (MIPyME) industriales mexicanas. Revista Venezolana de Información, Tecnología y Conocimiento, Vol. 10, No. 1. (85-104).

Superintendencia de la economía solidaria. (27 de Enero de 2017). Circular No. 04. http://www.supersolidaria.gov.co/sites/def ault/files/public/normativa/circular_sarlaft _2017.pdf. (Consultado: 30 de Agosto de 2018)

Yamakawa, P., \& Ostos, J. (2011). Relación entre innovación organizacional y desempeño organizacional. Universidad \& Empresa, 93-115.

Yong, L., Rivas, L., \& Chaparro, J. (2010). Modelo de aceptación tecnológica (TAM): un estudio de la influencia de la cultura nacional y del perfil del usuario en el uso de las TIC. Revista Innovar, Vol. 20, No. 36. (187-203).

Zapata, J., Arango, M., \& Adarme, W. (Diciembre de 2010). Herramientas tecnológicas al servicio de la gestión empresarial. Revista avances en sistemas e informática, Vol. 7 , No. 3. (87-101). (Consultado: 23 de 07 de 2018) 